Research Article

\title{
Time-Harmonic Response of an Elastic Pile in a Radially Inhomogeneous Poroelastic Medium
}

\author{
Xibin Li $\mathbb{D}$, Wenhui Xu, and Zhiqing Zhang \\ School of Landscape Architecture, Zhejiang A \& F University, Hangzhou 311300, Zhejiang, China \\ Correspondence should be addressed to Zhiqing Zhang; zhangzhiqing2000@163.com
}

Received 7 December 2020; Revised 11 January 2021; Accepted 22 January 2021; Published 9 February 2021

Academic Editor: Xinyu Xie

Copyright (@) 2021 Xibin Li et al. This is an open access article distributed under the Creative Commons Attribution License, which permits unrestricted use, distribution, and reproduction in any medium, provided the original work is properly cited.

\begin{abstract}
The time-harmonic response of an elastic pile embedded in a radially inhomogeneous poroelastic medium and subjected to a torsional loading is studied in the present article. In engineering practice, the surrounding soil may be weakened due to the disturbance effect caused by pile driving. To simulate the weakened surrounding soil, a boundary zone model with the complex shear modulus of the inner disturbed soil changing in a parabolic form along the radial direction is proposed. In view of the axissymmetric deformation of the surrounding soil under torsional load, the equation of motion of the saturated soil is solved in the cylindrical coordinate system. The vibration displacement and shear stress solutions for the inner disturbed soil are gained by expanding the displacement as a power series, and those for the outer undisturbed soil are obtained by solving the partial differential equation. By virtue of continuity conditions at the interface between inner and outer soil regions, the torsional impedance of the radially inhomogeneous soil is solved. Then, via the boundary and continuity conditions of the pile-soil system, the twist angle and torque of the pile are obtained in the frequency domain. Finally, selected numerical results are conducted to investigate the influence of the material damping, softening degree, and softening range of the inner soil on the distribution of the twist angle and torque of the pile along the depth direction.
\end{abstract}

\section{Introduction}

Foundations and pile foundations are commonly subjected to dynamic torsional load due to the eccentricity in applied lateral loads, and the corresponding dynamic analysis is of great importance for the dynamic foundation design. Over the past few decades, various theoretical methods (e.g., analytical, semianalytical, and numerical methods) were proposed to solve the vibration of piles or foundations in elastic/viscoelastic soil media. For instance, Novak and Howell [1] analyzed the torsional vibration characteristics of an end-bearing pile in a viscoelastic soil medium using an analytical method. $\mathrm{Wu}$ et al. [2] proposed the fictitious model to simulate the effect of the pile end soil and gained an analytical solution for the torsional dynamic response of a floating pile embedded in a layered soil. Wu et al. [3] investigated the vertical vibration characteristics of a variable impedance pile embedded in a multilayered soil using a quasi-analytical method. Militano and Rajapakse [4] proposed a semianalytical method to study the dynamic response of an elastic pile subjected to transient torsional and axial loads. Lin et al. [5], Pan et al. [6], and Zhang and Pan [7] studied the torsional and vertical vibration of rigid circular foundation over and buried in a transversely isotropic multilayered half-space via a semianalytical method. Besides, the numerical methods (e.g., finite element method, coupled finite element method-boundary element method) $[8,9]$ were also applied into the study on the torsional vibration of a pile embedded in a layered half-space.

Most of the above studies on piles had regarded soil as a radial homogenous elastic/viscoelastic media. Since Biot's pioneer work $[10,11]$ on the general theory of acoustic propagation in poroelastic medium, the dynamic response of the buried patch load [12-14], and the torsional vibration of pile or foundation in saturated soil were investigated in detail [15-19]. Besides, Zhao and Xiang [20] investigated the dynamic torsional response of pipe pile embedded in unsaturated soil. However, in practice, the soil adjacent to the 
pile may be compacted or loosened during pile installation [21], which results in soil properties changing continuously along the radial direction. That is to say, in this case, the soil is inhomogeneous in the radial direction. To approximately account for the inhomogeneity of the surrounding soil, Novak and Sheta [21] first proposed a weakened annular boundary zone model with an inner weakened massless region. In light of the limitation of this study, Veletsos and Dotson [22] further considered the mass of the soil, which can better reflect the inertia effect of the weakened soil. After that, various types of nonreflective boundary zone model containing inner disturbed region and outer undisturbed homogenous region were proposed, in which the shear modulus of the inner disturbed region changes continuously in the radial direction [23-25]. Moreover, Li and Gao [26] and Zhang et al. [27] studied, respectively, the vertical and torsional vibration characteristics of a pipe pile embedded in a radially inhomogeneous soil using the rigorous continuum soil model. However, these studies modeled the radially inhomogeneous soil as a viscoelastic medium. Based on Biot's poroelastodynamic theory, Li et al. $[28,29]$ derived quasi-analytical solutions for the dynamic torsional and vertical response of an end-bearing pile embedded in a radially inhomogeneous saturated soil.

To the best of our knowledge, an analytical solution for the dynamic torsional response of a floating pile in a radially inhomogeneous saturated soil is still unavailable. Therefore, the objective of the present study is to propose an analytical solution to study the time-harmonic torsional response of an elastic pile embedded in a radially inhomogeneous saturated soil. Through the numerical calculation, the influence of the material damping, softening degree, and softening range of the inner soil on the distribution of the twist angle and torque of the pile along the depth direction is investigated in detail. The present solution can be further served as benchmarks for the complicated and powerful numerical methods.

\section{Establishment of the Mathematical Model}

2.1. Geometric Model and Assumptions. Consider the dynamic torsional interaction between an elastic pile and a radially inhomogeneous poroelastic medium, as depicted in Figure 1 . The elastic pile is vertically with a uniform circular cross-section and subjected to a time-harmonic torsional load $T_{0} e^{i \omega t}$ acting at its top end. The length and radius of the pile are $H$ and $r_{0}$, respectively. In engineering practice, the poroelastic medium (i.e., saturated soil) adjacent to the pile would be compacted or loosened due to pile installation, which results in soil properties continuously changing in the radial direction. Hence, in order to describe the radial inhomogeneity of the soil in theory, the boundary zone model with nonreflective boundary is proposed, and the surrounding soil is then divided into an inner disturbed region of radial thickness $t_{m}$ and an outer semi-infinite undisturbed region (i.e., homogenous region). Furthermore, it is also assumed that the displacement and stress at the interface of the pile and inner soil region (and that of inner and outer soil regions) are continuous. Since the applied torsional load and

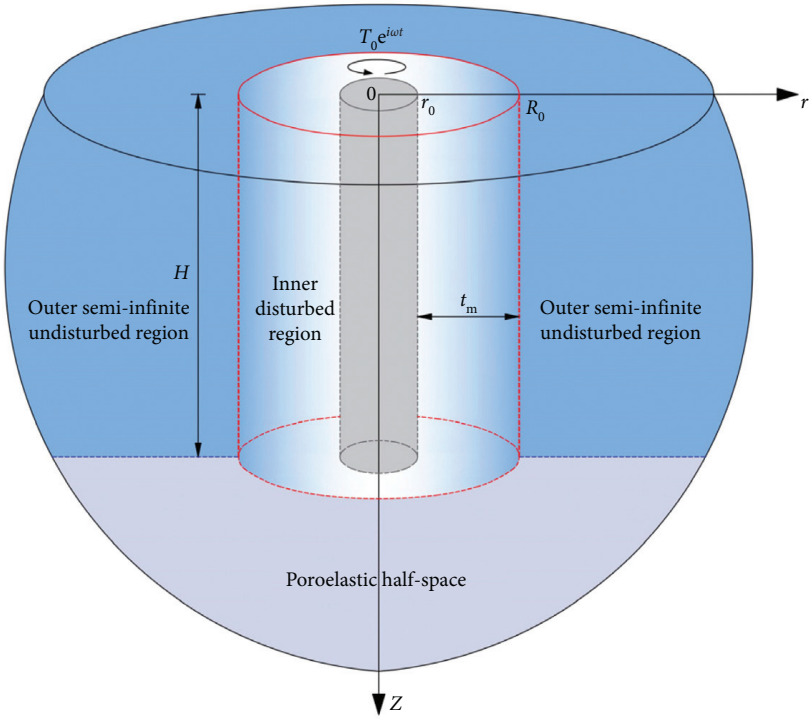

Figure 1: Geometric model of pile-soil dynamic interaction.

the deformation of the surrounding soil are axis-symmetric, we present and solve the pile-soil interaction problem in terms of the cylindrical coordinate system $(r, \theta, z)$, and the radially inhomogeneous poroelastic medium is within the positive $z$ domain. It should be also pointed out that under the action of the time-harmonic torsional load, the response is proportional to the factor $e^{i \omega t}$, which will be suppressed for brevity.

The geometry of the radially inhomogeneous soil is shown in Figure 2, in which the complex shear modulus of the inner disturbed soil is assumed to continuously change in a parabolic form proposed by Han and Sabin [24]. Therefore, the complex shear modulus of the soil in the inner and outer regions can be written in terms of the mathematical formulations as

$$
G_{s}^{*}(r)= \begin{cases}G_{s m}^{*}, & r=r_{0}, \\ G_{s 0}^{*} f(r), & r_{0} \leq r \leq R_{0}, \\ G_{s 0}^{*}, & r \geq R_{0},\end{cases}
$$

where $G_{s 0}^{*}=G_{s 0}\left(1+i D_{s 0}\right)$ and $G_{s m}^{*}=G_{s m}\left(1+i D_{s m}\right)$ are the complex shear modulus of the soil in the outer undisturbed region and the soil at the interface of the pile and soil; $D_{s 0}$ and $D_{s m}$ are, respectively, the corresponding soil damping coefficients at the same locations; $i=(-1)^{0.5} ; R_{0}$ is the radial distance from the interface of the two regions to the center of the pile; and $f(r)$ is a function reflecting the parabolic variation of the complex shear modulus of the soil in the inner region and considered to vary according to the following expression:

$$
f(r)=1-\beta^{2}\left(\frac{r-R_{0}}{r_{0}}\right)^{2}
$$

in which

$$
\beta^{2}=\frac{1-G R\left(1+i D_{s m}\right) /\left(1+i D_{s 0}\right)}{\left(t_{m} / r_{0}\right)^{2}}
$$




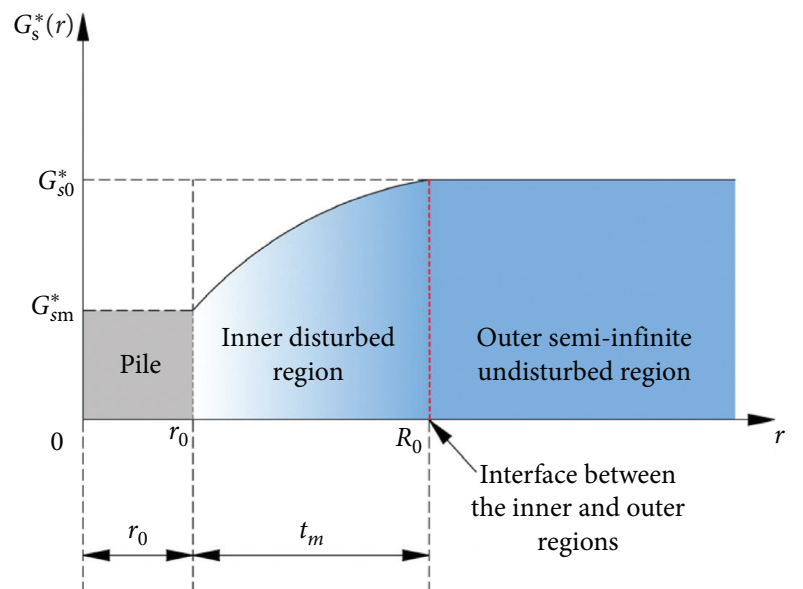

FIGURE 2: Geometry of the radially inhomogeneous saturated soil.

where $G R=G_{s m} / G_{s 0}$ reflects the disturbance (i.e., softening or hardening) degree of the inner soil and $t_{m}$ is the width of the inner disturbed region, which reflects the disturbance range of the inner soil. It is noted that $G R<1$ denotes the weakened inner soil and $G R>1$ denotes the strengthened inner soil.

2.2. Governing Equations. Based on Biot's general theory of acoustic propagation in the poroelastic medium $[10,11]$ and under the plane strain assumption of the soil [4], the dynamic equilibrium equation of the saturated soil subjected to torsional load can be written as

$$
\begin{aligned}
& G_{s}^{*}(r)\left[\frac{\partial^{2} u_{\theta}}{\partial r^{2}}+\frac{1}{r} \frac{\partial u_{\theta}}{\partial r}-\frac{u_{\theta}}{r^{2}}\right]+\frac{\mathrm{d} G_{s}^{*}(r)}{\mathrm{d} r}\left(\frac{\partial u_{\theta}}{\partial r}-\frac{u_{\theta}}{r}\right) \\
& \quad=-\rho \omega^{2} u_{\theta}-\rho_{f} \omega^{2} w_{\theta},
\end{aligned}
$$

where $u_{\theta}$ and $w_{\theta}$ are, respectively, the amplitude of the circumferential displacement of the soil skeleton and that of the pore fluid relative to the soil skeleton; $\rho=(1-\phi) \rho_{s}+\phi \rho_{f}$ is the density of the soil; $\rho_{s}$ and $\rho_{f}$ are the densities of soil skeleton and pore fluid, respectively; and $\phi$ is the porosity of the saturated soil.

The general dynamic equilibrium equation of the pore fluid for the poroelastic medium is proposed by Biot. Particularly for the saturated soil, the simplified dynamic equilibrium equation of the pore fluid can be written as [30]

$$
\begin{gathered}
i \omega \frac{\rho_{f m} g}{k_{d m}} w_{\theta}-\omega^{2} \rho_{f m} u_{\theta}-\omega^{2} \frac{\rho_{f m}}{\phi_{m}} w_{\theta}=0, \quad\left(r_{0} \leq r \leq R_{0}\right), \\
i \omega \frac{\rho_{f 0} g}{k_{d 0}} w_{\theta}-\omega^{2} \rho_{f 0} u_{\theta}-\omega^{2} \frac{\rho_{f 0}}{\phi_{0}} w_{\theta}=0, \quad\left(r \geq R_{0}\right),
\end{gathered}
$$

where $\rho_{m}=\left(1-\phi_{m}\right) \rho_{s m}+\phi_{m} \rho_{f m}$ and $\rho_{0}=\left(1-\phi_{0}\right) \rho_{s 0}+\phi_{0} \rho_{f 0}$ are the densities of soil in the disturbed and undisturbed regions, respectively; $k_{d j}, \rho_{s j}, \rho_{f j}$, and $\phi_{j}(j=m, 0)$ are the horizontal dynamic permeability coefficient containing the viscosity of the fluid, density of soil skeleton, density of pore fluid, and porosity of the saturated soil in the corresponding region, respectively; $g$ is the acceleration of gravity; and $\omega$ is the circular frequency of excitation.

For the convenience of subsequent derivation, equation (2) can be rewritten as

$$
f(\xi)=1-\left[\beta\left(\xi_{0}-\xi\right)\right]^{2},
$$

where

$$
\begin{gathered}
\xi=\frac{r}{r_{0}}, \\
\xi_{0}=\frac{R_{0}}{r_{0}} .
\end{gathered}
$$

Introducing $\mathrm{d} u_{\theta} / \mathrm{d} r=\mathrm{d} u_{\theta} /\left(r_{0} \mathrm{~d} \xi\right)$ and then substituting equations (5) and (6) into equation (4), yield

$$
\begin{array}{r}
f(\xi) \frac{\mathrm{d}^{2} u_{\theta}}{\mathrm{d} \xi^{2}}+\left[\frac{f(\xi)}{\xi}+\frac{\mathrm{d} f(\xi)}{\mathrm{d} \xi}\right] \frac{\mathrm{d} u_{\theta}}{\mathrm{d} \xi}-\left[\frac{f(\xi)}{\xi^{2}}+\frac{1}{\xi} \frac{\mathrm{d} f(\xi)}{\mathrm{d} \xi}+\lambda_{m}^{2}\right] u_{\theta}=0, \quad\left(1 \leq \xi \leq \xi_{0}\right), \\
\xi^{2} \frac{\mathrm{d}^{2} u_{\theta}}{\mathrm{d} \xi^{2}}+\xi \frac{\mathrm{d} u_{\theta}}{\mathrm{d} \xi}-\left(1+\lambda_{0}^{2} \xi^{2}\right) u_{\theta}=0, \quad\left(\xi \geq \xi_{0}\right),
\end{array}
$$

where

$$
\begin{aligned}
& \lambda_{j}=i \omega r_{0}\left[\frac{1}{G_{s j}^{*}}\left(\rho_{j}+\frac{\phi_{j} \rho_{f j} \omega}{i b_{j} / \rho_{f j}-\omega}\right)\right]^{(1 / 2)}, \\
& b_{j}=\frac{\phi_{j} \rho_{f j} g}{k_{d j}}, \quad(j=m, 0) .
\end{aligned}
$$

After introducing $x=\beta\left(\xi_{0}-\xi\right)$ and $\mathrm{d} u_{\theta} / \mathrm{d} \xi=-\beta \mathrm{d} u_{\theta} / \mathrm{d} x$, equation (8) can be rewritten in a much concise form

$$
\begin{aligned}
& \left(x^{2}-1\right) \frac{\mathrm{d}^{2} u_{\theta}}{\mathrm{d} x^{2}}+\left(\frac{x^{2}-1}{x-a}+2 x\right) \frac{\mathrm{d} u_{\theta}}{\mathrm{d} x} \\
& +\left[\frac{1-x^{2}}{(x-a)^{2}}-\frac{2 x}{x-a}+b\right] u_{\theta}=0, \quad\left(1 \leq \xi \leq \xi_{0}\right),
\end{aligned}
$$


where

$$
\begin{aligned}
& a=\beta \xi_{0}, \\
& b=\left(\frac{\lambda_{m}}{\beta}\right)^{2} .
\end{aligned}
$$

Given that the elastic pile undergoes forced torsional vibration, the dynamic equilibrium equation of the pile can be expressed as

$$
G_{p} \frac{\partial^{2}}{\partial z^{2}}\left[\varphi(z) e^{i \omega t}\right]+\frac{4 f(z) e^{i \omega t}}{r_{0}^{2}}=\rho_{p} \frac{\partial^{2}}{\partial t^{2}}\left[\varphi(z) e^{i \omega t}\right]
$$

where $G_{p}, \rho_{p}, r_{0}$, and $\varphi(z)$ are the shear modulus, density, radius, and the twist angle amplitude of the pile, respectively, and $f(z)$ denotes the amplitude of the contact traction along the pile-soil interface.

2.3. Boundary and Continuity Conditions. Considering that the displacement of the soil tends to zero at infinity, the boundary condition of the soil can be written as

$$
\left.u_{\theta}(r)\right|_{r \longrightarrow \infty}=0
$$

The continuity conditions of the inner and outer soil regions can be expressed as

$$
\begin{gathered}
\left.u_{\theta}(r)\right|_{r=R_{0-}}=\left.u_{\theta}(r)\right|_{r=R_{0+}}, \\
\left.\tau_{r \theta}(r)\right|_{r=R_{0-}}=\left.\tau_{r \theta}(r)\right|_{r=R_{0+}} .
\end{gathered}
$$

The boundary conditions of the pile are

$$
\begin{aligned}
\left.\frac{\mathrm{d} \varphi(z)}{\mathrm{d} z}\right|_{\mid z=0} & =-\frac{T_{0}}{G_{p} I_{p}}, \\
{\left[\frac{\mathrm{d} \varphi(z)}{\mathrm{d} z}+\frac{\varphi(z) k_{\mathrm{pb}}}{G_{p} I_{p}}\right]_{\mid z=H} } & =0,
\end{aligned}
$$

where $I_{p}=\left(\pi r_{0}^{4} / 2\right)$ denotes the polar moment of inertia of the pile and $k_{p b}$ is the supporting stiffness coefficient of the pile bottom. If the underlying half-space is elastic, $k_{p b}$ can be approximately taken as $\left(16 G_{s b} r_{0}^{3} / 3\right)$ [4] with $G_{s b}$ being the shear modulus of the underlying half-space. If the underlying half-space is rigid, $\varphi(z)$ should be equal to zero at the level $z=H$. Generally, we can fix $k_{p b}=\infty$ to simulate the rigid base case.

The continuity conditions at the pile-soil interface can be written as

$$
\begin{aligned}
& \left.u_{\theta}(r)\right|_{r=r_{0}}=\varphi(z) r_{0}, \\
& \left.\tau_{r \theta}(r)\right|_{r=r_{0}}=f(z) .
\end{aligned}
$$

\section{Solution of the Radially Inhomogeneous Soil Layer}

3.1. Solution of the Outer Soil Medium. For the saturated soil in the outer region, the solution of equation (9) can be expressed as

$$
u_{\theta}=C K_{1}\left(\lambda_{0} \xi\right)+D I_{1}\left(\lambda_{0} \xi\right), \quad\left(\xi \geq \xi_{0}\right),
$$

where $I_{1}\left(\lambda_{0} \xi\right)$ and $K_{1}\left(\lambda_{0} \xi\right)$ are the first-order modified Bessel functions of the first kind and the second kind, respectively. $C$ and $D$ are the constants determined by the boundary conditions.

It is noted from equation (19) that $D$ should vanish to zero to guarantee bounded displacement given in equation (14). Then, the amplitude of the circumferential displacement and shear stress of the soil in the outer region can be expressed as

$$
\begin{aligned}
u_{\theta} & =C K_{1}\left(\lambda_{0} \xi\right), \\
\tau_{r \theta} & =-\frac{1}{r_{0}} C G_{s 0}^{*} \lambda_{0} K_{2}\left(\lambda_{0} \xi\right) .
\end{aligned}
$$

3.2. Solution of the Inner Soil Medium. In order to solve equation (8), we express the circumferential displacement of the soil as the following infinite series:

$$
u_{\theta}=\sum_{m=0}^{\infty} A_{m} x^{m}
$$

It is noted that we only need a finite number of $m$ in equation (21) to ensure the accuracy of the results.

Substituting equation (21) into equation (8), after some algebraic operation (the detailed derivation for $A_{m}$ is listed in Appendix A), the recursive relation of coefficient $A_{m}$ can be written as

$$
\begin{cases}A_{m}=\frac{E_{1} A_{m-1}+E_{2} A_{m-2}+E_{3} A_{m-3}+E_{4} A_{m-4}}{m(m-1) a^{2}}, & m \geq 4, \\ A_{m}=\frac{2 a(1-b) A_{0}+a^{2}(b+2) A_{1}+6 a A_{2}}{6 a^{2}}, & m=3, \\ A_{m}=\frac{\left(a^{2} b+1\right) A_{0}+a A_{1}}{2 a^{2}}, & m=2,\end{cases}
$$

where

$$
\begin{aligned}
& E_{1}=(m-1)(2 m-3) a, \\
& E_{2}=(m-1)(m-2) a^{2}-(m-2)^{2}+b a^{2}+1, \\
& E_{3}=-a[(m-3)(2 m-3)+2(b-1)], \\
& E_{4}=(m-2)(m-4)+b-3 .
\end{aligned}
$$




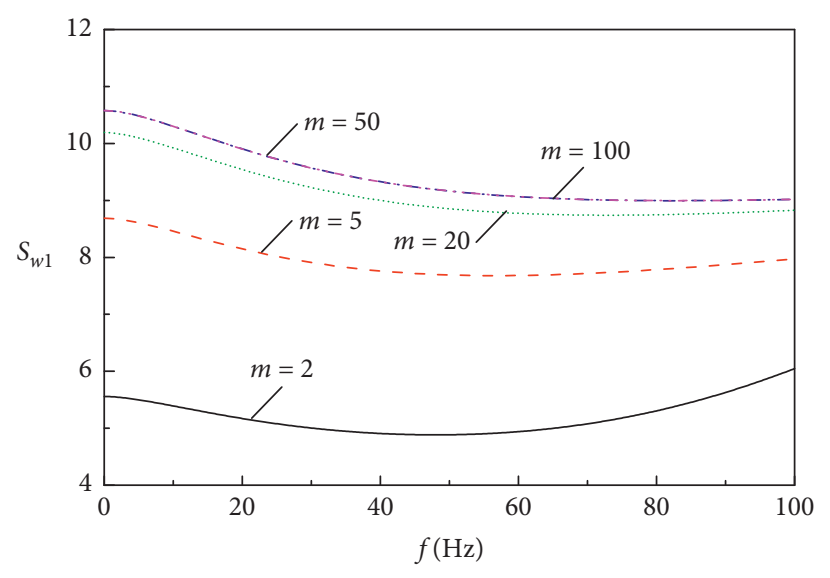

(a)

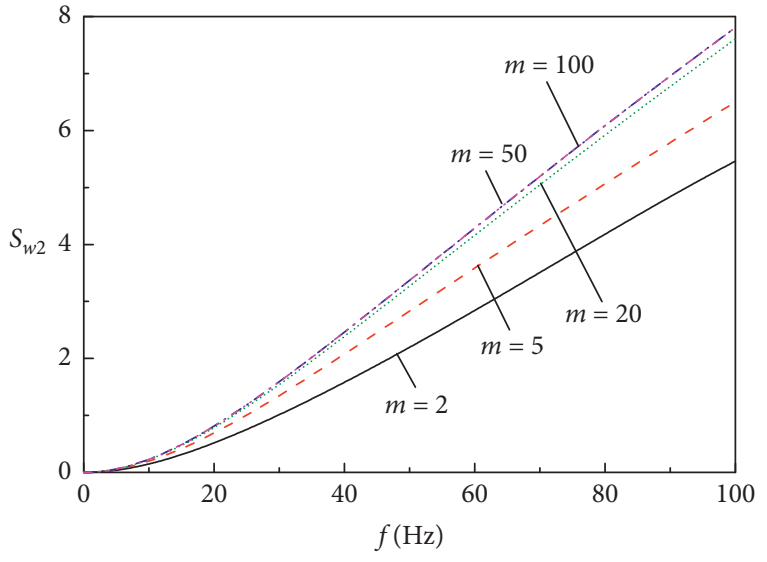

(b)

FIgURE 3: Influence of $m$ on the torsional impedance of the soil $(G R=0.5)$.

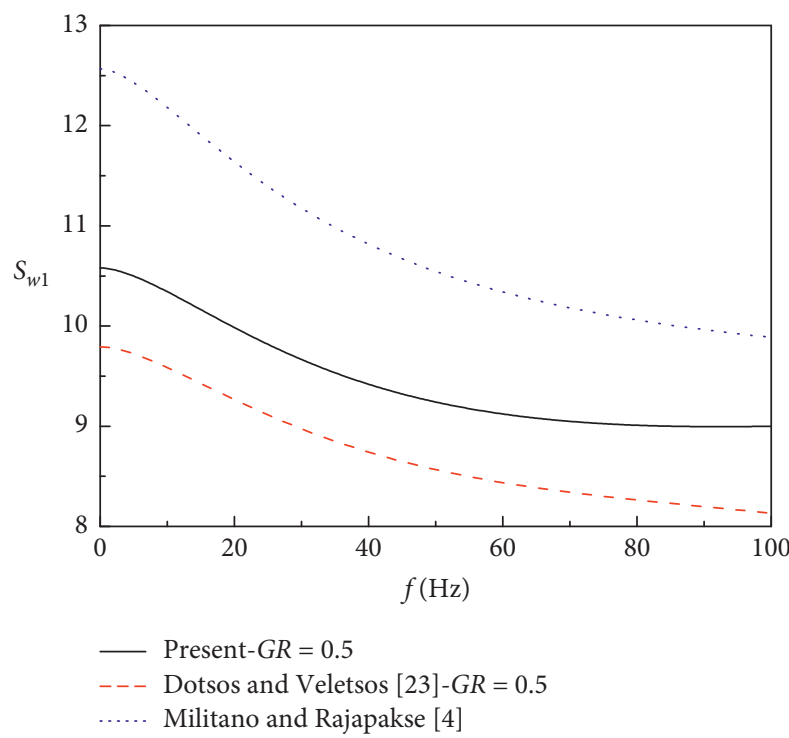

(a)

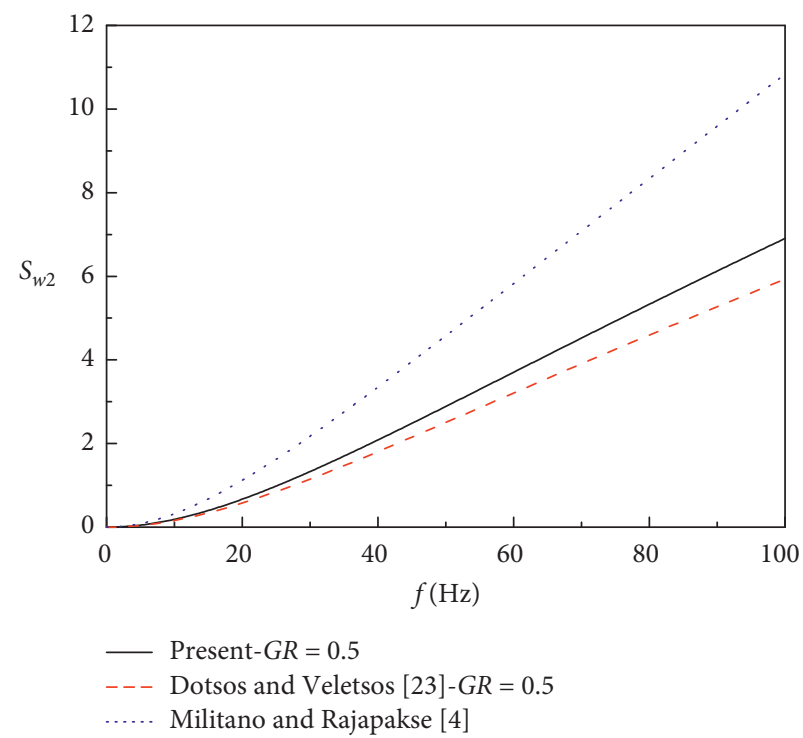

(b)

FIgURE 4: Comparison with the existing solutions for a purely elastic soil medium.

The amplitude of circumferential shear stress of the inner soil medium can be expressed as

$$
\tau_{r \theta}=-G_{s}^{*}(r)\left(\frac{\beta}{r_{0}} \frac{\mathrm{d} u_{\theta}}{\mathrm{d} x}+\frac{u_{\theta}}{r}\right)=-G_{s}^{*}(r)\left(\frac{\beta}{r_{0}} \sum_{m=1}^{\infty} A_{m} m x^{m-1}+\frac{1}{r} \sum_{m=0}^{\infty} A_{m} x^{m}\right) .
$$

Substituting equations (20), (21), and (24) into the continuity conditions given in equation (15), we have

$$
\begin{aligned}
& A_{0}=C K_{1}\left(\lambda_{0} \xi_{0}\right), \\
& A_{1}=\frac{\lambda_{0} \xi_{0} K_{2}\left(\lambda_{0} \xi_{0}\right)-K_{1}\left(\lambda_{0} \xi_{0}\right)}{\beta \xi_{0}} C .
\end{aligned}
$$

Then, the circumferential shear stress amplitude of the soil at the pile-soil interface can be expressed as

$$
\left.\tau_{r \theta}\right|_{r=r_{0}}=-\frac{G_{s m}^{*}}{r_{0}}\left(\beta \sum_{m=1}^{\infty} A_{m} m x_{0}^{m-1}+\sum_{m=0}^{\infty} A_{m} x_{0}^{m}\right)
$$

where $x_{0}=\left(1-G_{s m}^{*} / G_{s 0}^{*}\right)^{(1 / 2)}$. 


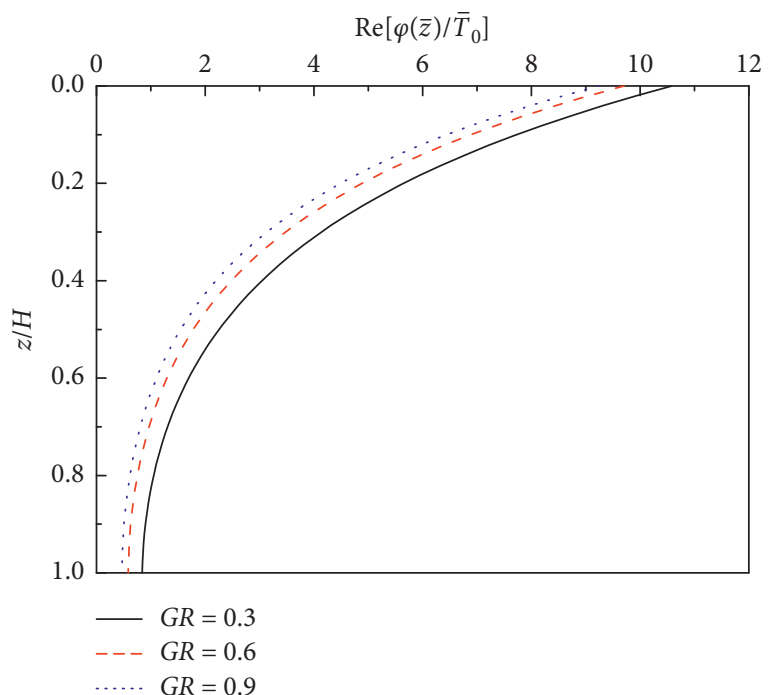

(a)

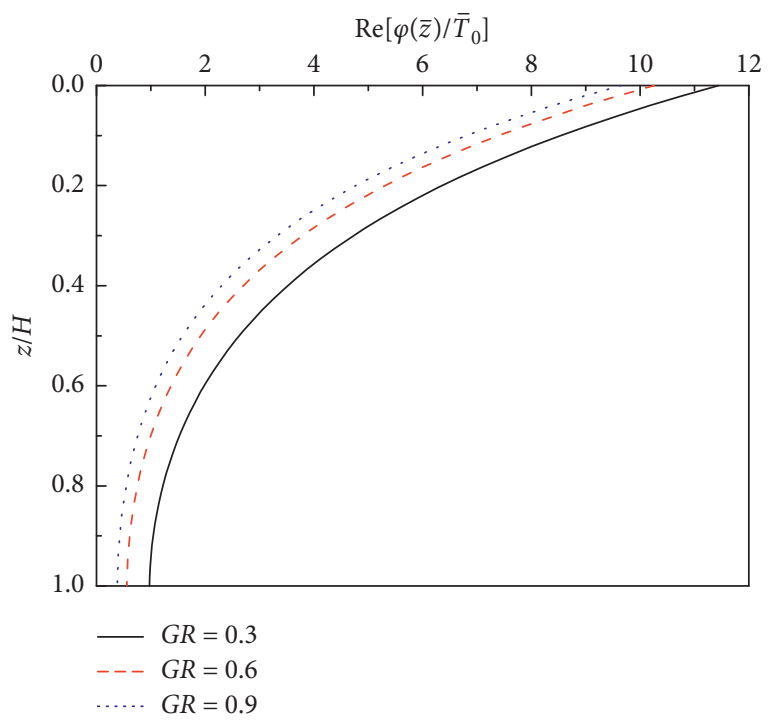

(c)

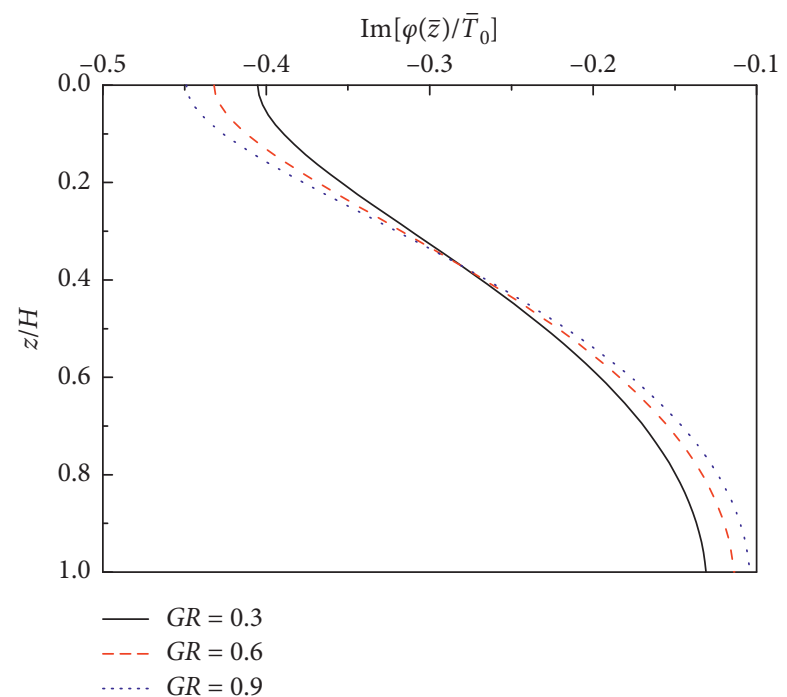

(b)

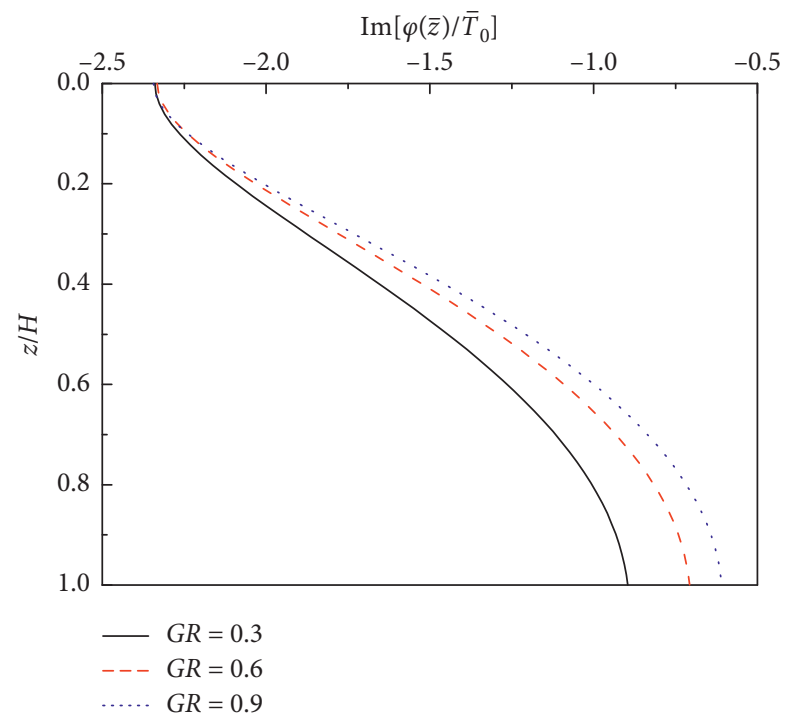

(d)

Figure 5: Influence of the softening degree of the inner soil on the distribution of normalized twist angle of the pile $(f=20 \mathrm{~Hz}$ in (a) and (b); $f=50 \mathrm{~Hz}$ in (c) and (d)).

The torsional impedance of the soil can be finally expressed as

$$
K_{\theta}=-\frac{\left.2 \pi r_{0}^{3} \tau_{r \theta}\right|_{r=r_{0}}}{\left.u_{\theta}\right|_{r=r_{0}}}=2 \pi r_{0}^{2} G_{s m}^{*}\left(\frac{\beta \sum_{m=1}^{\infty} A_{m} m x_{0}^{m-1}}{\sum_{m=0}^{\infty} A_{m} x_{0}^{m}}+1\right),
$$

where $K_{\theta}$ denotes the torsional impedance of the surrounding soil. It is worth noting that $A_{m}$ is a function of $C$; hence, the unknown constant $C$ can be automatically eliminated.

For the convenience of subsequent analysis, $K_{\theta}$ can be further expressed as the following form:

$$
K_{\theta}=G_{s 0} r_{0}^{2}\left(S_{w 1}+i S_{w 2}\right),
$$

where $S_{w 1}$ and $S_{w 2}$ denote, respectively, the stiffness and damping part of the torsional impedance of the radially inhomogeneous saturated soil.

\section{Solution of the Elastic Pile}

Combining equations (17) and (21), we have

$$
\sum_{m=0}^{\infty} A_{m} x_{0}^{m}=\varphi(z) r_{0} .
$$

Then, the circumferential shear stress amplitude of the soil at the pile-soil interface can be further expressed as

$$
\left.\tau_{r \theta}\right|_{r=r_{0}}=-\frac{K_{\theta}}{2 \pi r_{0}^{2}} \varphi(z) .
$$




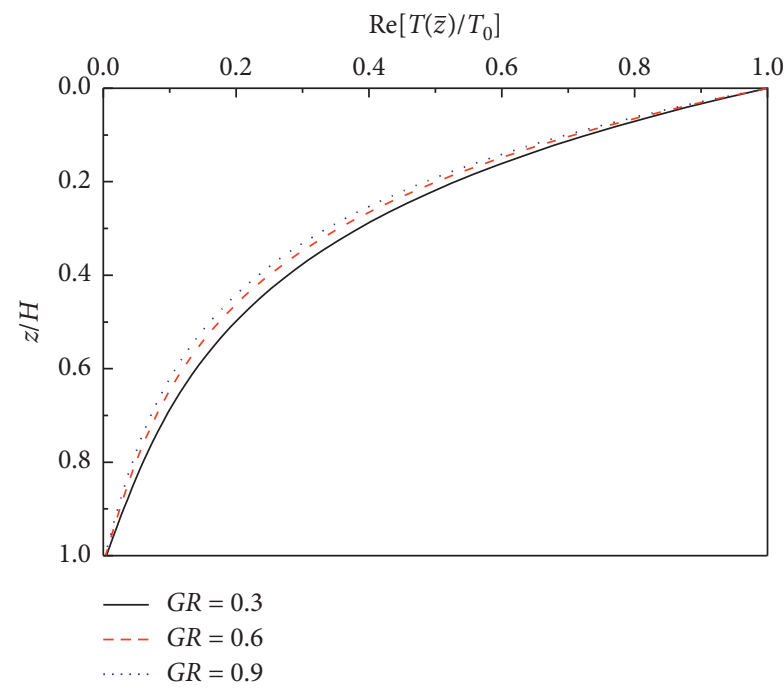

(a)

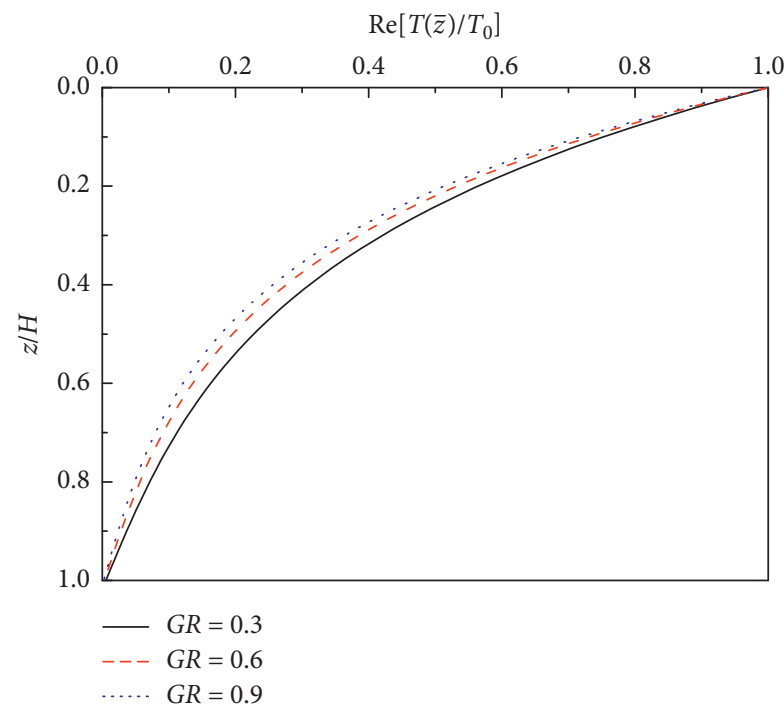

(c)

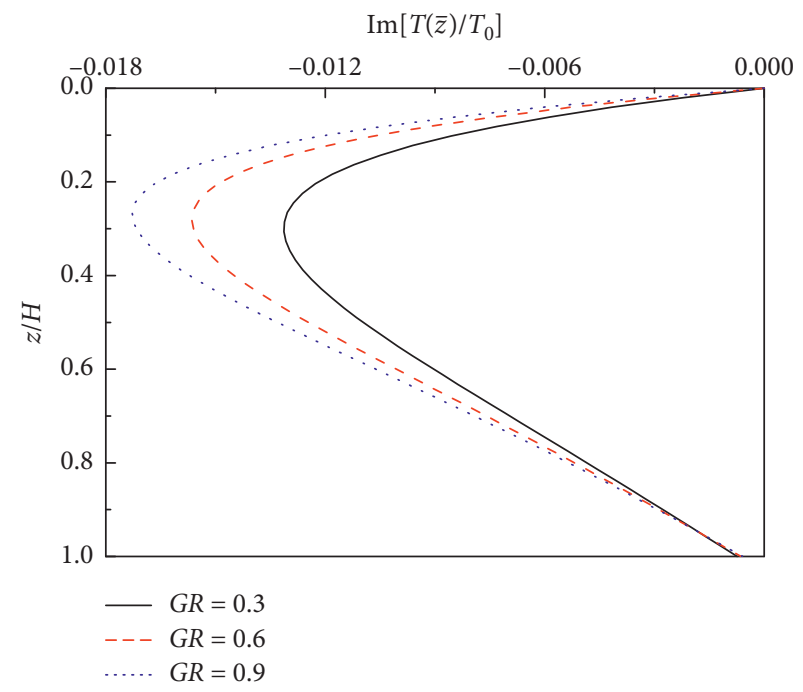

(b)

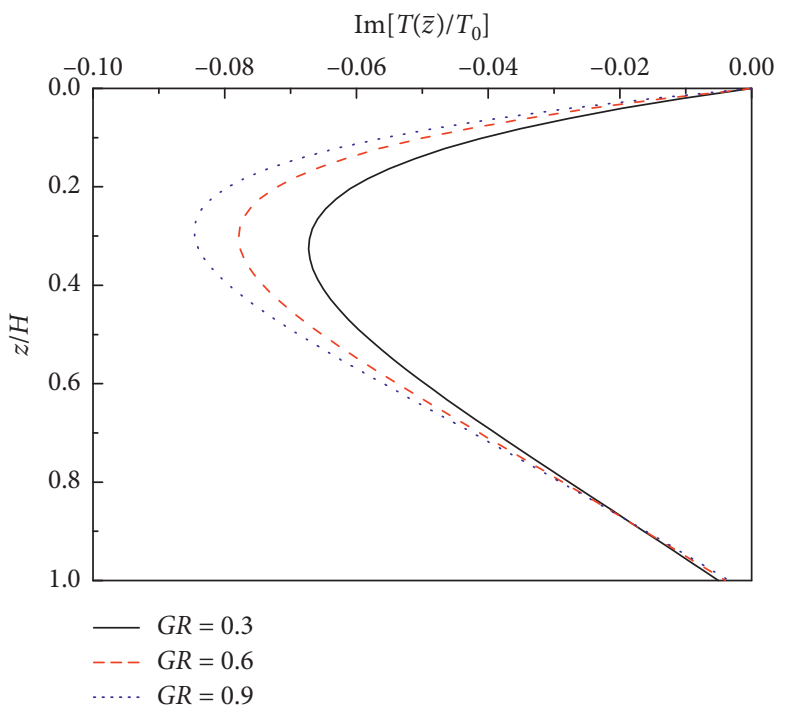

(d)

Figure 6: Influence of the softening degree of the inner soil on the distribution of normalized torque of the pile $(f=20 \mathrm{~Hz}$ in (a) and (b); $f=50 \mathrm{~Hz}$ in (c) and (d)).

Substituting equation (30) into equation (18), the governing equation for the torsional vibration of the pile under time-harmonic torsional load can be further written as

$$
\frac{\mathrm{d}^{2} \varphi(z)}{\mathrm{d} z^{2}}+\left[-\frac{2 K_{\theta}}{G_{p} \pi r_{0}^{4}}+\frac{\rho_{p} \omega^{2}}{G_{p}}\right] \varphi(z)=0 .
$$

Solving the ordinary differential equation (31), we have

$$
\varphi(z)=\alpha_{1} \cos (\gamma z)+\alpha_{2} \sin (\gamma z)
$$

in which

$$
\gamma=\left[-\frac{2 K_{\theta}}{G_{p} \pi r_{0}^{4}}+\frac{\rho_{p} \omega^{2}}{G_{p}}\right]^{(1 / 2)},
$$

where $\alpha_{1}$ and $\alpha_{2}$ are the constants determined by the boundary conditions.

Substituting the boundary conditions of the pile given in equation (16) into equation (33) results in

$$
\begin{aligned}
& \alpha_{1}=-\frac{T_{0}}{G_{p} I_{p} \gamma \tan (\gamma H-\delta)}, \\
& \alpha_{2}=-\frac{T_{0}}{G_{p} I_{p} \gamma},
\end{aligned}
$$

where $\delta=\arctan \left[\bar{k}_{p b} /(\gamma H)\right]$ denotes the phase angle and $\bar{k}_{p b}=\left(k_{p b} H /\left(G_{p} I_{p}\right)\right)$ denotes the dimensionless pile bottom supporting coefficient.

The torque of the pile body can be further expressed as 


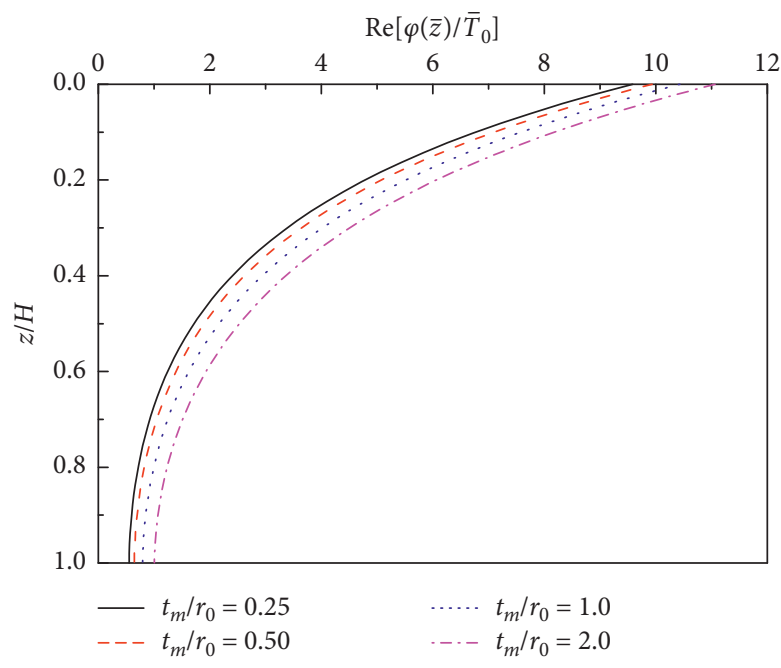

(a)

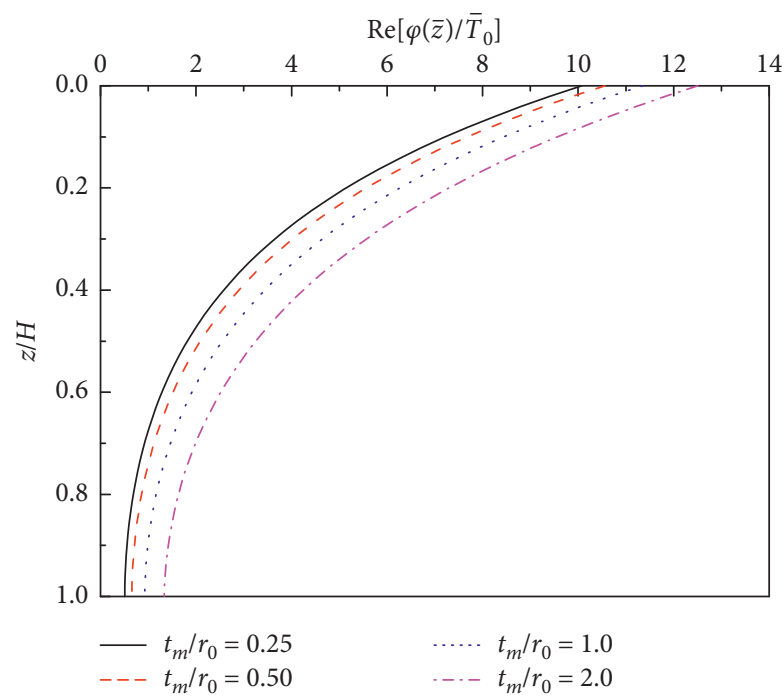

(c)

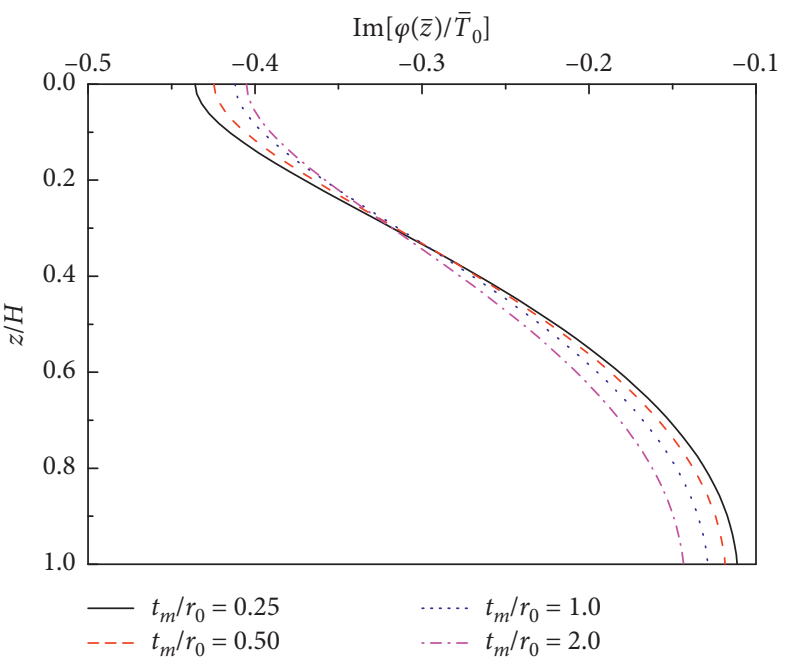

(b)

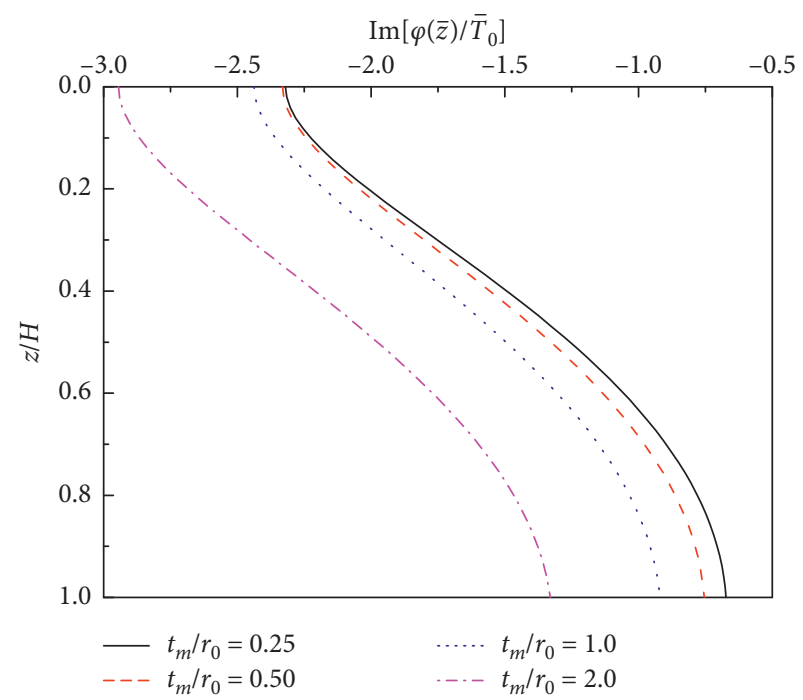

(d)

FIGURE 7: Influence of the softening range of the inner soil on the distribution of normalized twist angle of the pile $(G R=0.5 ; f=20 \mathrm{~Hz}$ in $(\mathrm{a})$ and (b); $f=50 \mathrm{~Hz}$ in (c) and (d)).

$$
T(z)=-G_{p} I_{p} \frac{\mathrm{d} \varphi(z)}{\mathrm{d} z}=-G_{p} I_{p} \gamma\left[-\alpha_{1} \sin (\gamma z)+\alpha_{2} \cos (\gamma z)\right] .
$$

\section{Numerical Analysis and Discussion}

The influence of the soil radial heterogeneity on the torsional impedance of the soil and torsional complex stiffness at the pile top has received detailed investigation in the past studies $[22-25,28]$. Therefore, the effect of the soil radial heterogeneity on the distribution of the twist angle and torque of the pile body will be mainly analyzed in the present study. In addition, it is found from the past studies [23-25, 28] that the strengthened inner soil can improve the ability of the pile-soil system to resist dynamic torsional deformation, which is safe for the dynamic foundation design in general cases. Hence, the emphasis of the present study is to analyze the influence of the weakened inner soil on the torsional vibration characteristics of the pile-soil system. In the calculation, it is assumed that the porosity, permeability coefficient, density of the soil skeleton, and density of the pore fluid of the soil in the inner region are equal to the corresponding parameters of the soil in the outer region. Unless otherwise specified, the parameters of the saturated soil and pile used in calculation are $G_{s 0}=G_{s b}=20 \mathrm{MPa}, \rho_{s m}=\rho_{s 0}=$ $2650 \mathrm{~kg} / \mathrm{m}^{3}, \rho_{f m}=\rho_{f 0}=1000 \mathrm{~kg} / \mathrm{m}^{3}, \phi_{m}=\phi_{0}=0.4, k_{d m}=k_{d 0}=$ $10^{-7} \mathrm{~m} / \mathrm{s}, \quad D_{s m}=D_{s 0}=0, \quad \rho_{p}=2500 \mathrm{~kg} / \mathrm{m}^{3}, \quad G_{p}=12.1 \mathrm{GPa}$, $H=10 \mathrm{~m}, r_{0}=0.3 \mathrm{~m}, t_{m} / r_{0}=0.5$.

In order to solve the torsional vibration of the inner disturbed soil, an infinite series is used to express the circumferential displacement of the soil. Hence, the number of calculation items (i.e., value of $m$ ) has great influence on the reliability and accuracy of the present solution. The influence 


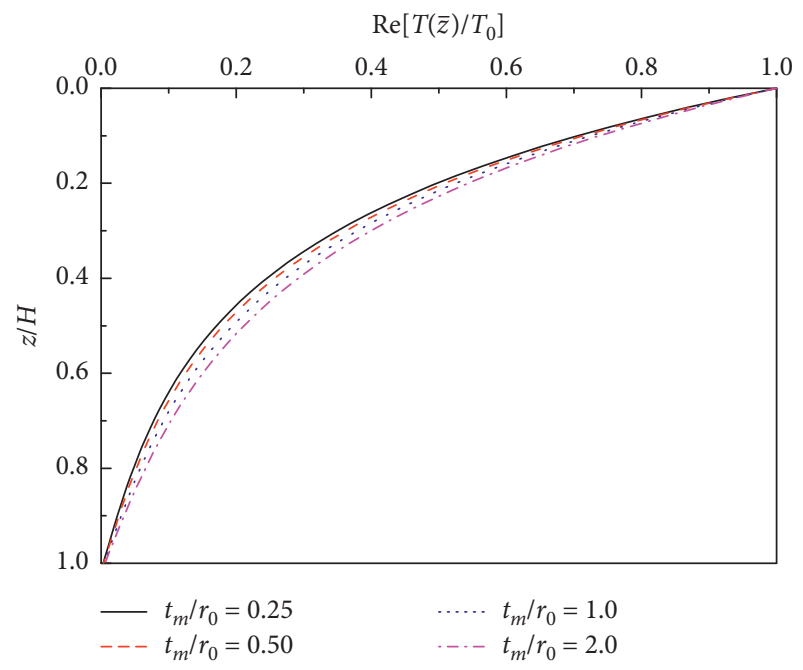

(a)

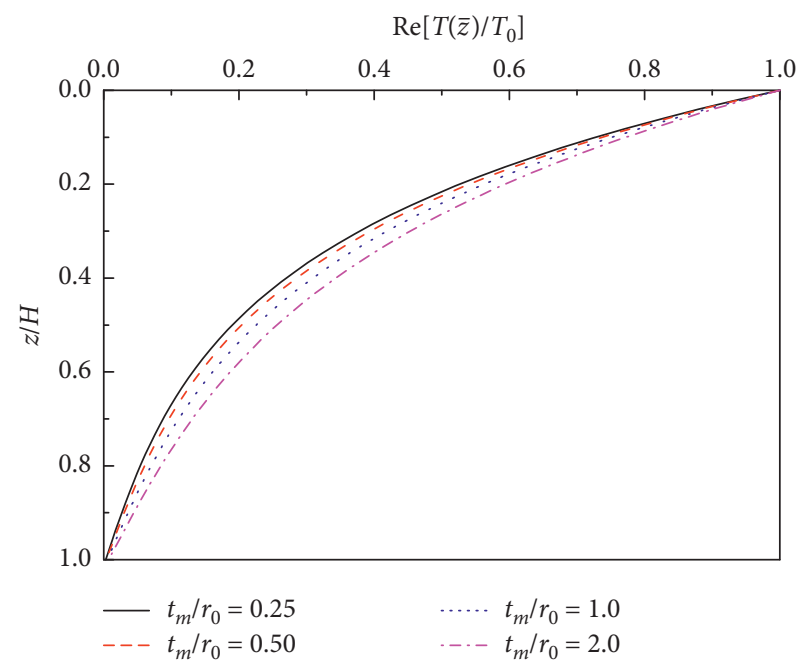

(c)

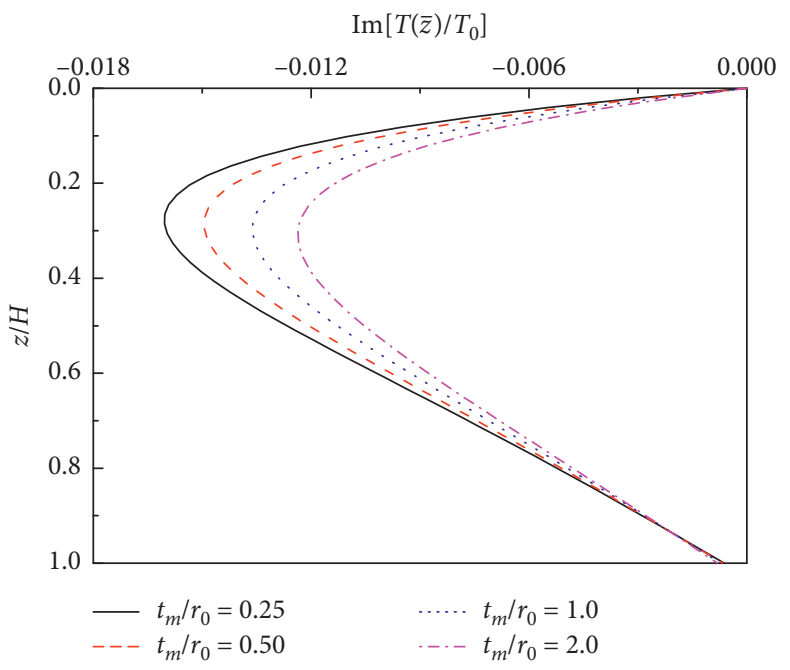

(b)

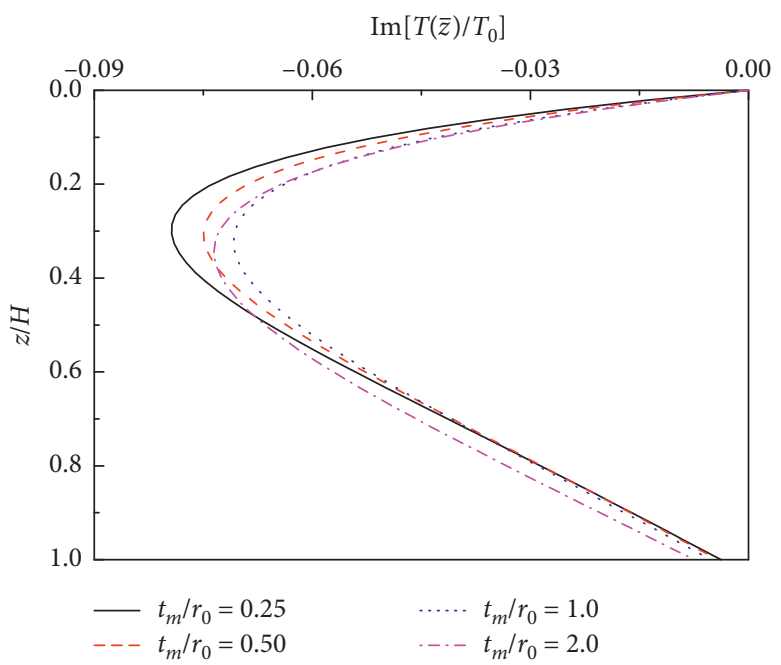

(d)

FIGURE 8: Influence of the softening range of the inner soil on the distribution of normalized torque of the pile $(G R=0.5 ; f=20 \mathrm{~Hz}$ in (a) and (b); $f=50 \mathrm{~Hz}$ in (c) and (d)).

of the value of $m$ on the reliability and accuracy on the soil torsional impedance is shown in Figure 3. It can be seen that when $m$ is small (e.g., $m=2,5,20$ ), the infinite series has not converged to a stable value, and the curves corresponding to different $m$ are quite different. However, the soil torsional impedance will be convergent when $m$ is greater than 50. In order to ensure the accuracy of calculation, $m=100$ are used in subsequent calculations.

Figure 4 shows the comparison of the reduced purely elastic soil medium (i.e., $\rho_{f}=0$ ) of the present solution with other existing solutions. It can be seen from Figure 4 that, in the low-frequency range, the real and imaginary parts of the present solution and Dotsos and Veletsos's solution [23] are lower than those of Militano and Rajapakse's homogenous solution [4]. It is noted that the present solution assumes that the shear modulus of the inner soil continuously changes in a parabolic form along the radial direction, while the solution by Dotsos and Veletsos [23] assumed that the shear modulus of the inner soil continuously changes in an exponential form. This indicates that regardless of the changing form of the shear modulus of the weakened soil in the inner region, its torsional impedance is necessarily lower than that of the undisturbed soil (i.e., homogenous soil). It can be also observed from Figure 4 that the real and imaginary parts of the present solution are larger than those of Dotsos and Veletsos [23]. This phenomenon indicates that the changing form of the shear modulus of the weakened soil has a great influence on the impedance of the soil, although the values of $G R$ and $t_{m} / r_{0}$ remain constants.

Figure 5 depicts the influence of the softening degree (GR) on the distribution of the normalized twist angle of the pile for different frequencies. It should be pointed out that smaller $G R$ means a larger softening degree of the inner soil. The normalized twist angle is defined as $\left(\varphi(\bar{z}) / \bar{T}_{0}\right)$, where $\bar{T}_{0}=\left(T_{0} r_{0} /\left(G_{p} I_{p}\right)\right)$. $\operatorname{Re}[]$ and $\operatorname{Im}[]$ denote, respectively, the real and imaginary parts of the corresponding physical 


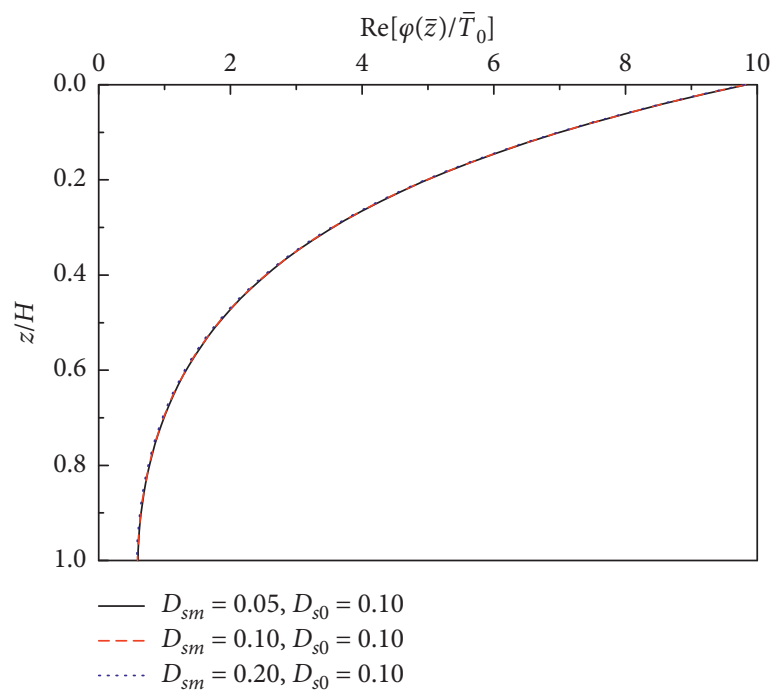

(a)

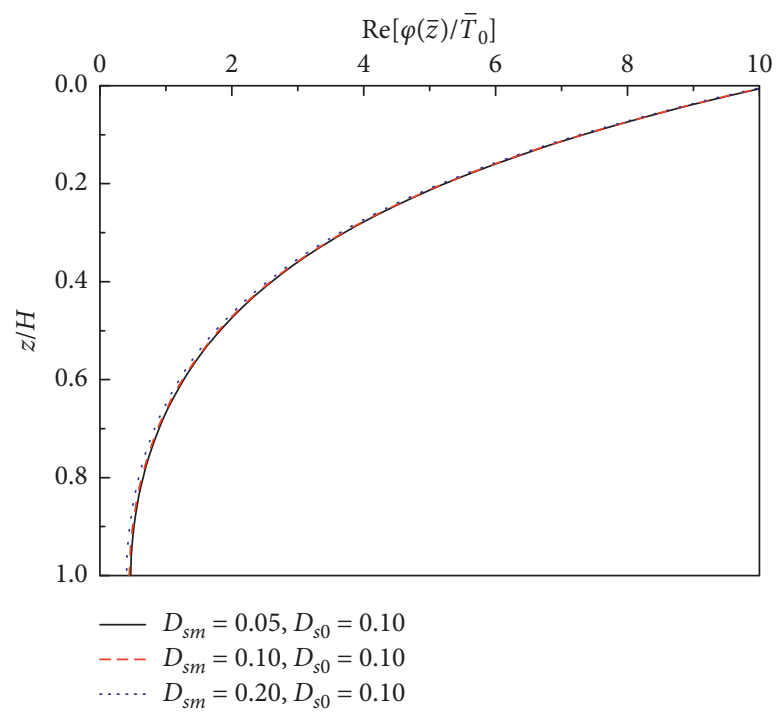

(c)

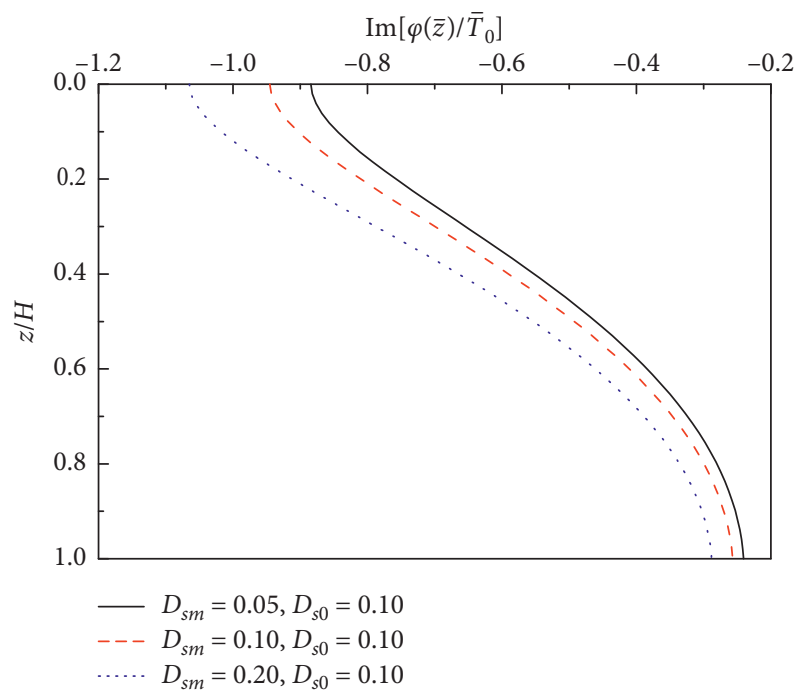

(b)

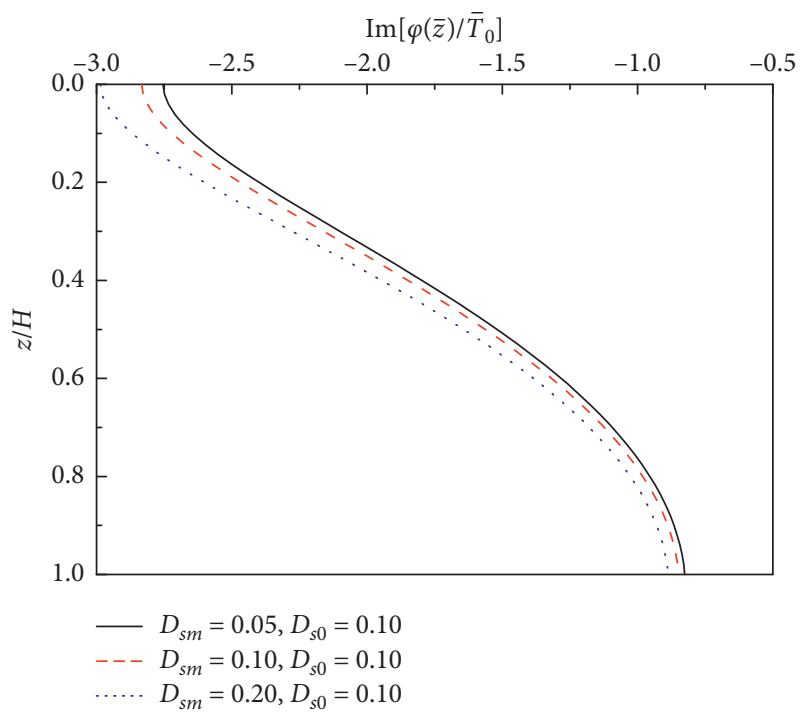

(d)

Figure 9: Influence of the material damping of the inner soil on the distribution of normalized twist angle of the pile $(G R=0.5 ; f=20 \mathrm{~Hz}$ in (a) and (b); $f=50 \mathrm{~Hz}$ in (c) and (d)).

quantity. It can be observed from Figure 5 that the softening degree has a marked influence on the distribution of the twist angle of the pile along the depth direction. The following trends can also be observed from Figure 5: (1) the real part dominates the twist angle distribution, and it gradually decreases along the depth direction. (2) The real part of the twist angle of the pile top and the pile body increases with decreasing GR. This indicates that the twist angle of the pile will increase with the increase of softening degree. (3) The changing trend of the imaginary part is relatively complicated. When $f=20 \mathrm{~Hz}$, there exists a turning point. Above this point, the absolute value of the imaginary part increases with increasing GR. Below this point, the imaginary part decreases with increasing GR. However, when $f=50 \mathrm{~Hz}$, the absolute value of the imaginary part decreases with increasing $G R$.
Figure 6 shows the influence of the softening degree $(G R)$ on the distribution of the normalized torque of the pile for different frequencies. The normalized torque of the pile is defined as $\left(T(\bar{z}) / T_{0}\right)$. It can be seen from Figure 6 that $G R$ has a marked influence on the distribution of normalized torque along the depth direction. The real part also dominates the distribution of torque. At a fixed depth, the real part increases with the decrease of $G R$, while the absolute value of the imaginary part decreases with the decrease of $G R$. This indicates that the higher the softening degree of the inner soil, the smaller the load borne by the soil and accordingly the greater the load borne by the pile. It can be also seen from Figure 6 that the imaginary part has a peak at the critical depth. Above the critical depth, the absolute value of the imaginary part increases with increasing $z$. Below the 


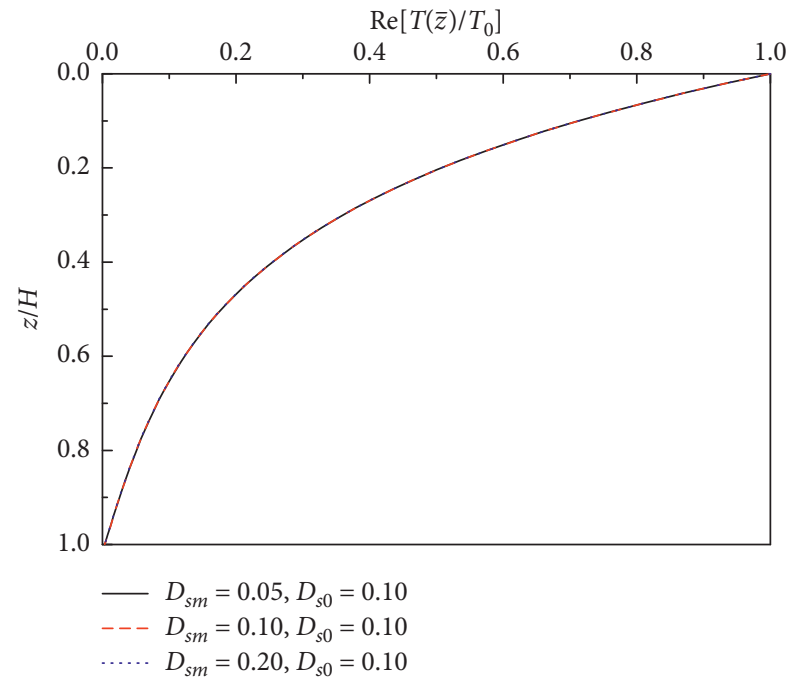

(a)

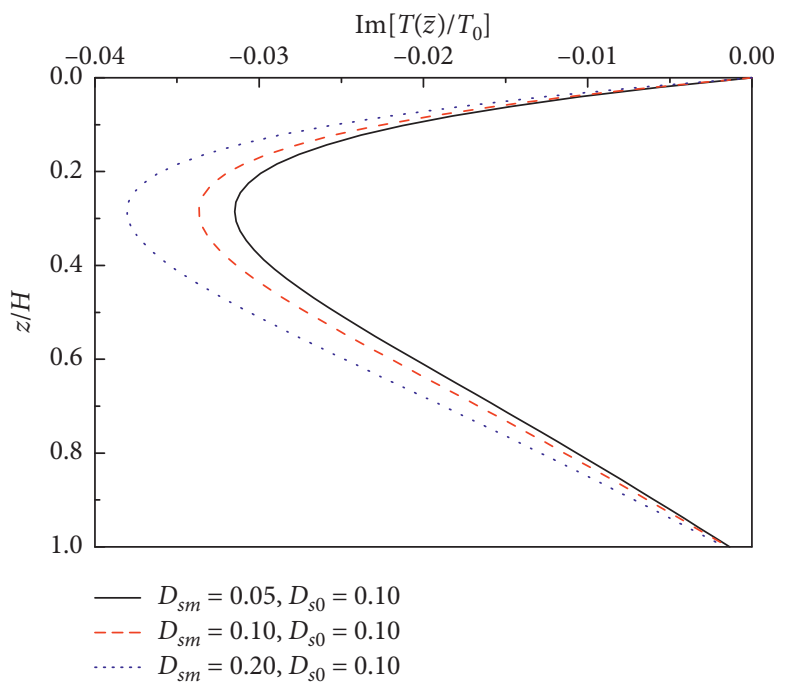

(b)

FIGURE 10: Influence of the material damping of the inner soil on the distribution of normalized torque of the pile $(G R=0.5 ; f=20 \mathrm{~Hz}$ in (a) and (b)).

critical depth, the absolute value of the imaginary part decreases as $z$ increases.

Figure 7 describes the influence of the softening range $\left(t_{m} / r_{0}\right)$ on the distribution of the normalized twist angle of the pile for different frequencies. It can be observed from Figure 7 that the real part increases with increasing softening range. This indicates that a large softening range is associated with the large twist angle of the pile with this trend being more remarkable when the frequency is relatively high (e.g., $f=50 \mathrm{~Hz}$ ). The changing trend of the imaginary part is also dependent on the frequency. At different frequencies, the changing trend is totally different.

Figure 8 shows the influence of the softening range $\left(t_{m} / r_{0}\right)$ on the distribution of the normalized torque of the pile for different frequencies. It can be seen from Figure 8 that the real part increases with the increase of $t_{m} / r_{0}$ (especially when $z /$ $H=0.3-0.6$ ) for different frequencies with the increase being more pronounced as frequency increases. When $f=20 \mathrm{~Hz}$, the absolute value of the imaginary part decreases with increasing $t_{m} / r_{0}$. However, the changing trend of the imaginary part is more complicated when $f=50 \mathrm{~Hz}$.

Figure 9 depicts the influence of the material damping of the inner soil on the distribution of the normalized twist angle of the pile for different frequencies. It can be seen from Figure 9 that the material damping of the inner soil has a slight influence on the distribution of the real part only when the frequency is relatively high (e.g., $f=50 \mathrm{~Hz}$ ). However, the material damping of the inner soil has an obvious influence on the imaginary part. The absolute value of the imaginary part increases with the increase of the material damping of the inner soil.

Figure 10 shows the influence of the material damping of the inner soil on the distribution of the normalized torque of the pile. It can be seen from Figure 10 that the material damping of the inner soil has negligible influence on the real part of the torque when $f=20 \mathrm{~Hz}$. However, the material damping of the inner soil has an obvious influence on the imaginary part. The absolute value of the imaginary part increases with the increase of the material damping of the inner soil. Through further calculation, the same changing trend is observed for $f=50 \mathrm{~Hz}$ and the corresponding result is not presented here.

\section{Conclusions}

In this paper, we solve the dynamic response of an elastic pile subjected to time-harmonic torsional load and embedded in a radially inhomogeneous saturated soil with its complex shear modulus in the inner region continuously changing in a parabolic form along the radial direction. According to Biot's poroelastodynamic theory and introducing a power series to express the circumferential displacement of the saturated soil in the inner region, the analytical solution of the twist angle and torque of the pile is obtained. Selected numerical results are carried out to analyze the influence of the material damping, softening degree, and softening range of the inner soil on the distribution of twist angle and torque along the depth direction. The main features observed from the numerical studies in the low-frequency range are concluded as follows:

(1) The changing form of the shear modulus of the weakened soil has marked influence on the torsional impedance of the soil. That is to say, a different changing form of the shear modulus of the weakened soil corresponds to a different torsional impedance of the soil, although the values of $G R$ and $t_{m} / r_{0}$ remain fixed constants.

(2) The softening degree and softening range of the inner soil have a marked influence on the distribution of the twist angle of the pile along the depth direction. The real part dominates the twist angle distribution. 
At fixed depth, the real part increases with the increase of softening degree (i.e., decreasing GR) and softening range (i.e., increasing $t_{m} / r_{0}$ ). The changing trend of the imaginary part is relatively complicated and is different for different frequencies.

(3) The softening degree and softening range of the inner soil have a marked influence on the distribution of torque of the pile along the depth direction. The real part also dominates the torque distribution and increases with the increase of softening degree and softening range.

(4) The absolute value of the imaginary parts of the twist angle and torque increases with the increase of the material damping of inner soil. However, the material damping of the inner soil has negligible influence on the corresponding real parts.

\section{Appendix}

\section{A. Detailed Derivation Procedure for $A_{m}$}

The first- and second-order derivatives of the power series given in equation (21) can be expressed as

$$
\begin{aligned}
\frac{\mathrm{d} u_{\theta}}{\mathrm{d} x} & =\sum_{m=1}^{\infty} m A_{m} x^{m-1}, \\
\frac{\mathrm{d}^{2} u_{\theta}}{\mathrm{d} x^{2}} & =\sum_{m=2}^{\infty} m(m-1) A_{m} x^{m-2} .
\end{aligned}
$$

Substituting equation (A.1) into equation (11) results in

$$
\left(x^{2}-1\right) \sum_{m=2}^{\infty} m(m-1) A_{m} x^{m-2}+\left(\frac{x^{2}-1}{x-a}+2 x\right) \sum_{m=1}^{\infty} m A_{m} x^{m-1}+\left[\frac{1-x^{2}}{(x-a)^{2}}-\frac{2 x}{x-a}+b\right] \sum_{m=0}^{\infty} A_{m} x^{m}=0
$$

Multiplying both sides of equation (A.2) by $(x-a)^{2}$ and where simplifying the power series, we have

$$
P+\sum_{m=2}^{\infty} Q A_{m} x^{m-2}=0
$$

$$
\begin{aligned}
P= & b A_{1} x^{3}+\left[(b-3) A_{0}-a(3+2 b) A_{1}\right] x^{2}+\left[2 a(1-b) A_{0}+\left(a^{2} b+2 a^{2}\right) A_{1}\right] x+\left(a^{2} b+1\right) A_{0}+a A_{1}, \\
Q= & {[m(m+2)+b-3] x^{4}+[-a m(2 m+3)+2 a(1-b)] x^{3} } \\
& +\left[a^{2} m(m+1)-m^{2}+a^{2} b+1\right] x^{2}+a(2 m-1) m x-a^{2} m(m-1) .
\end{aligned}
$$

The second term in the left side of equation (A.3) can be further written as

$$
\begin{aligned}
\sum_{m=2}^{\infty} \mathrm{Q} & A_{m} x^{m-2} \\
= & \sum_{m=6}^{\infty}[(m-4)(m-2)+b-3] A_{m-4} x^{m-2}+\sum_{m=5}^{\infty}[-a(m-3)(2 m-3)-2 a(b-1)] A_{m-3} x^{m-2} \\
& +\sum_{m=4}^{\infty}\left[a^{2}(m-2)(m-1)-(m-2)^{2}+a^{2} b+1\right] A_{m-2} x^{m-2} \\
& +\sum_{m=3}^{\infty} a(2 m-3)(m-1) A_{m-1} x^{m-2}+\sum_{m=2}^{\infty}-a^{2} m(m-1) A_{m} x^{m-2} .
\end{aligned}
$$


Combining equations (A.3), (A.4), and (A.6), we have

$$
\begin{gathered}
\sum_{m=2}^{\infty} E_{4} A_{m-4} x^{m-2}+\sum_{m=2}^{\infty} E_{3} A_{m-3} x^{m-2}+\sum_{m=2}^{\infty} E_{2} A_{m-2} x^{m-2} \\
+\sum_{m=2}^{\infty} E_{1} A_{m-1} x^{m-2}+\sum_{m=2}^{\infty}-a^{2} m(m-1) A_{m} x^{m-2}=0
\end{gathered}
$$

where

$$
\begin{aligned}
& E_{1}=(m-1)(2 m-3) a, \\
& E_{2}=(m-1)(m-2) a^{2}-(m-2)^{2}+b a^{2}+1, \\
& E_{3}=-a[(m-3)(2 m-3)+2(b-1)], \\
& E_{4}=(m-2)(m-4)+b-3 .
\end{aligned}
$$
holds:

In order to satisfy equation (A.7), the following relation

$$
E_{4} A_{m-4}+E_{3} A_{m-3}+E_{2} A_{m-2}+E_{1} A_{m-1}-a^{2} m(m-1) A_{m}=0 .
$$

For $m \geq 4$, the recurrence formula of $A_{m}$ can be expressed as

$$
A_{m}=\frac{E_{1} A_{m-1}+E_{2} A_{m-2}+E_{3} A_{m-3}+E_{4} A_{m-4}}{m(m-1) a^{2}} .
$$

It is noted that $A_{m}=0$ when $m<0$. Then, substituting $m=2$ and $m=3$ into equation (A.10), we have

$$
\begin{aligned}
& A_{2}=\frac{a A_{1}+\left(a^{2} b+1\right) A_{0}}{2 a^{2}}, \\
& A_{3}=\frac{6 a A_{2}+a^{2}(2+b) A_{1}+2 a(b-1) A_{0}}{6 a^{2}} .
\end{aligned}
$$

\section{Data Availability}

The data in Figures 3-10 used to support the findings of this study are available from the corresponding author upon request.

\section{Conflicts of Interest}

The authors declare that they have no conflicts of interest.

\section{Acknowledgments}

This research was supported by the National Natural Science Foundation of China (Grant no. 52078467), the Natural Science Foundation of Zhejiang Province (Grant no. LHZ21E090001), and Research and Development Fund of Zhejiang A\&F University (Grant no. 2020FR052).

\section{References}

[1] M. Novak and J. F. Howell, "Torsional vibration of pile foundations," Journal Of Geotechnical Engineering Division, vol. 103, no. GT4, pp. 271-285, 1977.
[2] W. Wu, H. Liu, M. H. El Naggar, G. Mei, and G. Jiang, "Torsional dynamic response of a pile embedded in layered soil based on the fictitious soil pile model," Computers and Geotechnics, vol. 80, pp. 190-198, 2016.

[3] W. B. Wu, X. L. Xu, H. Liu, C. L. Fang, B. Dou, and R. Z. Liang, "Vertical vibration characteristics of a variable impedance pile embedded in layered soil," Mathematical Problems in Engineering, vol. 2017, Article ID 1794950, 11 pages, 2017.

[4] G. Militano and R. K. N. D. Rajapakse, "Dynamic response of a pile in a multi-layered soil to transient torsional and axial loading," Géotechnique, vol. 49, no. 1, pp. 91-109, 1999.

[5] G. Lin, Z. Han, and J. Li, "An efficient approach for dynamic impedance of surface footing on layered half-space," Soil Dynamics and Earthquake Engineering, vol. 49, pp. 39-51, 2013.

[6] E. Pan, H. Liu, and Z. Zhang, "Vertical and torsional vibrations of a rigid circular disc on a transversely isotropic and layered half-space with imperfect interfaces," Soil Dynamics and Earthquake Engineering, vol. 113, pp. 442-453, 2018.

[7] Z. Zhang and E. Pan, "Vertical and torsional vibrations of an embedded rigid circular disc in a transversely isotropic multilayered half-space," Engineering Analysis with Boundary Elements, vol. 99, pp. 157-168, 2019.

[8] W. M. Liu and M. Novak, "Dynamic response of single piles embedded in transversely isotropic layered media," Earthquake Engineering and Structural Dynamics, vol. 23, no. 11, pp. 1239-1257, 1994.

[9] L. G. Tham, Y. K. Cheung, and Z. X. Lei, “Torsional dynamic analysis of single piles by time-domain BEM," Journal of Sound and Vibration, vol. 174, no. 4, pp. 505-519, 1994.

[10] M. A. Biot, "Theory of propagation of elastic waves in a fluidsaturated porous solid. i. low-frequency range," The Journal of the Acoustical Society of America, vol. 28, no. 2, pp. 168-178, 1956.

[11] M. A. Biot, "Mechanics of deformation and acoustic propagation in porous media," Journal of Applied Physics, vol. 33, no. 4, pp. 1482-1498, 1962.

[12] K. Sahebkar and M. Eskandari-Ghadi, "Time-harmonic response of saturated porous transversely isotropic half-space under surface tractions," Journal of Hydrology, vol. 537, pp. 61-73, 2016.

[13] J. Liang, M. Wu, and Z. Ba, “Three-dimensional dynamic Green's functions for transversely isotropic saturated halfspace subjected to buried loads," Engineering Analysis with Boundary Elements, vol. 108, pp. 301-320, 2019.

[14] Z. Zhang and E. Pan, "Time-harmonic response of transversely isotropic and layered poroelastic half-spaces under general buried loads," Applied Mathematical Modelling, vol. 80 , pp. $426-453,2020$.

[15] Y. Cai, G. Chen, C. Xu, and D. Wu, "Torsional response of pile embedded in a poroelastic medium," Soil Dynamics and Earthquake Engineering, vol. 26, no. 12, pp. 1143-1148, 2006.

[16] G. Wang, W. Ge, X. Pan, and Z. Wang, "Torsional vibrations of single piles embedded in saturated medium," Computers and Geotechnics, vol. 35, no. 1, pp. 11-21, 2008.

[17] G. Chen, Y. Cai, F. Liu, and H. Sun, "Dynamic response of a pile in a transversely isotropic saturated soil to transient torsional loading," Computers and Geotechnics, vol. 35, no. 2, pp. 165-172, 2008.

[18] L. Chen and G. Wang, "Torsional vibrations of elastic foundation on saturated media," Soil Dynamics and Earthquake Engineering, vol. 22, no. 3, pp. 223-227, 2002.

[19] Z. Zhang and E. Pan, "Vertical vibration of a rigid circular disc embedded in a transversely isotropic and layered poroelastic 
half-space," Engineering Analysis with Boundary Elements, vol. 118, pp. 84-95, 2020.

[20] F. Y. Zhao and P. Xiang, "Analysis of Rotary vibration of rigid friction pipe pile in unsaturated soil," Mathematical Problems in Engineering, vol. 2020, Article ID 280757, 12 pages, 2020.

[21] M. Novak and M. Sheta, "Approximate approach to contact problems of piles," Proceedings Of the Geotechnical Engineering Division, pp. 53-79, American Society of Civil Engineering National Convention, Miami, FL, USA, 1980.

[22] A. S. Veletsos and K. W. Dotson, "Vertical and torsional vibration of foundations in inhomogeneous media," Journal of Geotechnical Engineering, vol. 114, no. 9, pp. 1002-1021, 1988.

[23] K. W. Dotson and A. S. Veletsos, "Vertical and torsional impedances for radially inhomogeneous viscoelastic soil layers," Soil Dynamics and Earthquake Engineering, vol. 9, no. 3, pp. 110-119, 1990.

[24] Y. C. Han and G. C. W. Sabin, "Impedances for radially inhomogeneous viscoelastic soil media," Journal of Engineering Mechanics, vol. 121, no. 9, pp. 939-947, 1995.

[25] M. H. El Naggar, "Vertical and torsional soil reactions for radially inhomogeneous soil layer," Structural Engineering and Mechanics, vol. 10, no. 4, pp. 299-312, 2000.

[26] Z. Li and Y. Gao, "Effects of inner soil on the vertical dynamic response of a pipe pile embedded in inhomogeneous soil," Journal of Sound and Vibration, vol. 439, pp. 129-143, 2019.

[27] Y. P. Zhang, X. Y. Yang, W. B. Wu, M. H. El Naggar, G. S. Jiang, and R. Z. Liang, "Torsional complex impedance of pipe pile considering pile installation and soil plug effect," Soil Dynamics and Earthquake Engineering, vol. 131, Article ID 106010, 2020.

[28] Z. Li, Y. Gao, and K. Wang, "Torsional vibration of an end bearing pile embedded in radially inhomogeneous saturated soil," Computers and Geotechnics, vol. 108, pp. 117-130, 2019.

[29] Z. Y. Li, Y. F. Gao, and K. H. Wang, "Vertical vibration of an end bearing pile interacting with the radially inhomogeneous saturated soil," Ocean Engineering, vol. 219, Article ID 108009, 2020.

[30] O. C. Zienkiewicz, C. T. Chang, and P. Bettess, "Drained, undrained, consolidating and dynamic behaviour assumptions in soils," Géotechnique, vol. 30, no. 4, pp. 385-395, 1980. 